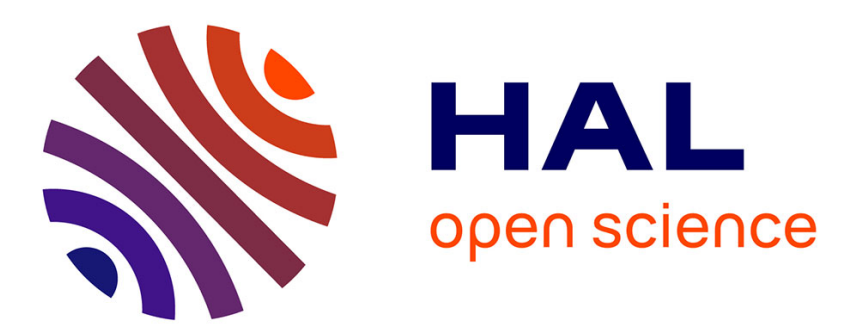

\title{
Mise en évidence de la modulation linéaire de l'intensité de la lumière due à l'anisotropie de la biréfringence magnétique linéaire
}

\author{
Tran Kkanh Vien, J.P. Jamet, H. Le Gall, R. Krishnan
}

\section{- To cite this version:}

Tran Kkanh Vien, J.P. Jamet, H. Le Gall, R. Krishnan. Mise en évidence de la modulation linéaire de l'intensité de la lumière due à l'anisotropie de la biréfringence magnétique linéaire. Revue de Physique Appliquée, 1975, 10 (6), pp.393-397. 10.1051/rphysap:01975001006039300 . jpa-00243934

HAL Id: jpa-00243934

https://hal.science/jpa-00243934

Submitted on 1 Jan 1975

HAL is a multi-disciplinary open access archive for the deposit and dissemination of scientific research documents, whether they are published or not. The documents may come from teaching and research institutions in France or abroad, or from public or private research centers.
L'archive ouverte pluridisciplinaire HAL, est destinée au dépôt et à la diffusion de documents scientifiques de niveau recherche, publiés ou non, émanant des établissements d'enseignement et de recherche français ou étrangers, des laboratoires publics ou privés. 


\title{
MISE EN ÉVIDENCE DE LA MODULATION LINÉAIRE DE L'INTENSITÉ DE LA LUMIËRE DUE A L'ANISOTROPIE DE LA BIRÉFRINGENCE MAGNÉTIQUE LINÉAIRE
}

\author{
TRAN KHANH VIEN, J. P. JAMET, H. LE GALL \\ Equipe de Magnétisme et d'Optique des Solides, C. N. R. S., 92190 Meudon-Bellevue, France \\ R. KRISHNAN \\ Laboratoire de Magnétisme, C. N. R. S., 92190 Meudon-Bellevue, France
}

\begin{abstract}
Résumé. - Nous mettons en évidence une modulation linéaire de l'intensité d'un faisceau lumineux par les effets $\mathrm{M}$. O. du $2^{\mathrm{e}}$ ordre, basée sur l'anisotropie de la B. M. L. Nous présentons les résultats obtenus en utilisant des cristaux magnétiques de symétrie cubique $\left(\mathrm{R}_{3} \mathrm{Fe}_{5} \mathrm{O}_{12}\right)$ et de symétrie rhomboédrique $\left(\alpha-\mathrm{Fe}_{2} \mathrm{O}_{3}\right)$.
\end{abstract}

Abstract. - A linear modulation of light induced by the second order magneto-optical interaction, and due to the anisotropy of the L. M. B. in magnetic crystals is shown. Experimental results are obtained by using cubic magnetic crystals $\left(\mathrm{R}_{3} \mathrm{Fe}_{5} \mathrm{O}_{12}\right)$ and a rhomboedral crystal $\left(\alpha-\mathrm{Fe}_{2} \mathrm{O}_{3}\right)$.

1. Introduction. - Jusqu'à présent, on obtient une modulation linéaire de l'intensité d'un faisceau lumineux en utilisant les effets $\mathrm{M}$. O. du $1^{\mathrm{er}}$ ordre ou effet Faraday [1, 2]. Nous montrons, dans cet article, que les effets $\mathbf{M}$. O. du $2^{\mathbf{e}}$ ordre peuvent donner la même modulation grâce à une propriété magnétooptique nouvelle des cristaux magnétiques. Cette propriété est l'anisotropie de la biréfringence magnétique linéaire (B. M. L.) ou de l'effet Cotton-Mouton $[3,4,6]$.

Dans la partie 2.1 , nous présentons en détail le principe de cette modulation de lumière et les résultats obtenus lorsque le cristal magnéto-optique est de symétrie cubique (cas des grenats ferrimagnétiques $\mathrm{R}_{3} \mathrm{Fe}_{5} \mathrm{O}_{12}$ ). Dans la partie 2.2, nous présentons les résultats obtenus avec l'hématite $\alpha-\mathrm{Fe}_{2} \mathrm{O}_{3}$, cristal de symétrie rhomboédrique.

2. Modulation magnéto-optique utilisant l'anisotropie de l'effet Cotton-Mouton. - Dans les cristaux magnétiques transparents, il a été démontré $[3,4]$ que les effets M. O. du $2^{\mathrm{e}}$ ordre sont décrits par un tenseur M. O. symétrique de rang 4 , le tenseur M. O. $f_{i j k l}$. La forme de ce tenseur dépend de la symétrie du cristal magnéto-optique :

2.1 Cristaux Cubiques (CAS Des GRenats Ferri-
MAGNÉTIQUES $\mathrm{R}_{3} \mathrm{Fe}_{5} \mathrm{O}_{12}$ ). - On montre [4] que, dans le cas des cristaux cubiques, les éléments du tenseur $f_{i j k l}$ ne dépendent que de 3 coefficients $\mathbf{M}$. O. indépendants : les coefficients $f_{11}, f_{12}$ et $f_{44}$.

Dans la géométrie Cotton-Mouton (propagation lumineuse perpendiculaire à l'aimantation du cristal), les effets $\mathrm{M}$. O. du $2^{\mathrm{e}}$ ordre se manifestent par une biréfringence magnétique linéaire (B. M. L.). Le déphasage Cotton-Mouton $\varphi_{\mathrm{CM}}$ qui caractérise cette B. M. L. est alors anisotrope, c'est-à-dire dépend de l'orientation de l'aimantation par rapport aux axes cristallographiques du cristal [4]. Ainsi dans le cas d'une propagation perpendiculaire à un plan (100), lorsque l'orientation de $\mathbf{M}$ varie dans ce plan, on montre que $\varphi_{\mathrm{CM}}$ vérifie la relation suivante [4] :

$\varphi_{\mathrm{CM}}=\frac{1}{2 \sqrt{\varepsilon_{\mathrm{r}}}} q_{0} \sqrt{4 f_{44}^{2}+\Delta f\left(\Delta f+4 f_{44}\right) \cos ^{2} 2} \alpha M^{2}$

où $\alpha$ est l'angle que fait $\mathbf{M}$ avec la direction [010] du plan ; $\Delta f$ est le coefficient d'anisotropie M. O. défini par $\Delta f=f_{11}-f_{12}-2 f_{44}$. La figure 1 illustre l'évolution de $\varphi_{\mathrm{CM}}$ en fonction de $\alpha$ dans ce cas pour un cristal de $\mathrm{Y}_{3} \mathrm{Fe}_{5} \mathrm{O}_{12}$. De même on montre que dans le cas d'une propagation perpendiculaire à un plan (110), $\varphi_{\mathrm{CM}}$ aura la forme suivante [4] :

$$
\varphi_{\mathrm{CM}}=\frac{1}{2 \sqrt{\varepsilon_{\mathrm{r}}}} q_{0} \sqrt{\left\{2 f_{44}-\frac{\Delta f}{2}\left(3 \sin ^{2} \alpha-1\right)\right\}^{2}+4 f_{44} \Delta f \sin ^{2} \alpha\left(3 \sin ^{2} \alpha-1\right)} M^{2}
$$




$$
\phi_{C M}=\frac{1}{2} q_{0} M^{2} \sqrt{4 f_{44}^{2}+\Delta f\left(\Delta f+4 f_{44}\right) \cos ^{2} 2 \propto}
$$

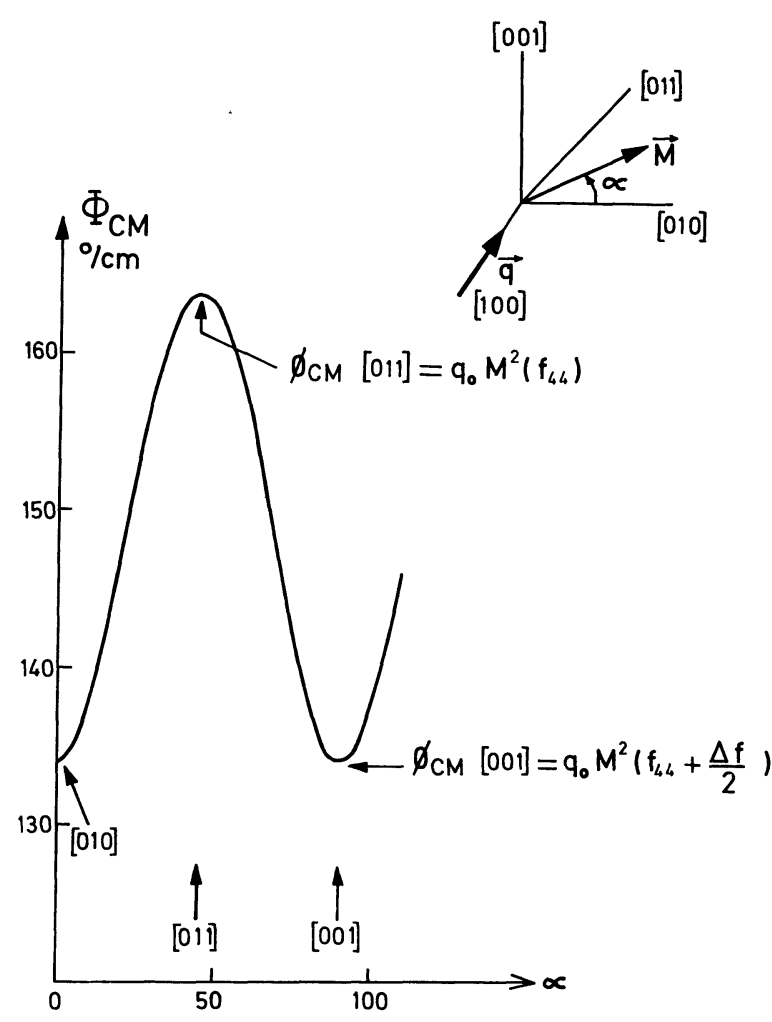

FIG. 1. - Evolution de la B. M. L. dans le cas d'une propagation perpendiculaire à un plan (100). (Valeurs numériques de $\varphi_{\mathrm{CM}}$ correspondant au grenat $\mathrm{Y}_{3} \mathrm{Fe}_{5} \mathrm{O}_{12}$ pour $\lambda=1,15 \mu, T=300 \mathrm{~K}$ et $H_{0}=5 \mathrm{kOe}$.)

$\alpha$ est l'angle que fait $\mathbf{M}$ avec une direction [110] du plan. La figure 2 montre l'évolution de $\varphi_{\mathrm{CM}}$ en fonction de $\alpha$ dans ce cas.

Comme nous voyons sur les figures 1 et 2 , l'anisotropie de la B. M. L. se manifeste par une variation de $\varphi_{\mathrm{CM}}$ avec $\alpha$ et cette anisotropie est caractérisée par le coefficient $\Delta f$.

On observe que, pour certaines valeurs bien définies de $\alpha, \varphi_{\mathrm{CM}}$ est une fonction linéaire de $\alpha$ en première approximation.

On définit la pente $K$ de la B. M. L. par :

$$
K=\left(\frac{\mathrm{d} \varphi_{\mathrm{CM}}}{\mathrm{d} \alpha}\right)_{\alpha_{0}} .
$$

Cette pente est une fonction des coefficients M. O. caractéristiques du cristal ainsi que de l'orientation de $\mathbf{M}$ par rapport aux axes cristallographiques du milieu.

Nous utilisons cette propriété pour obtenir une modulation linéaire d'un faisceau lumineux. En effet sur la figure 2 , on voit que, autour de certaines valeurs de $\alpha$, une variation $\Delta \alpha(t)$ de l'orientation de $\mathbf{M}$ dans le plan provoque une variation proportionnelle de $\Delta \varphi_{\mathrm{CM}}(t)$ de $\varphi_{\mathrm{CM}}$; or nous avons montré [5] que le montage de la figure 3 permet de transformer cette

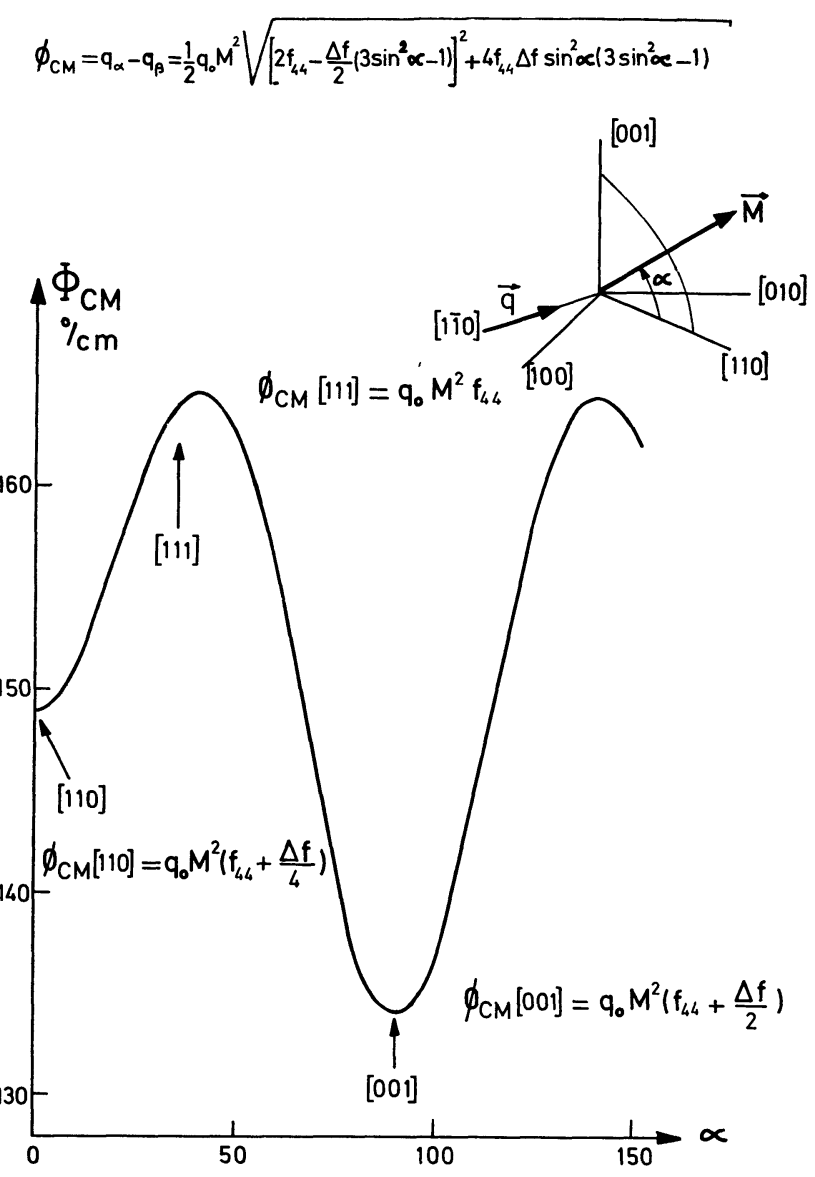

Fig. 2. - Evolution de la B. M. L. dans le cas d'une propagation perpendiculaire à un plan (110) (même matériau que pour la figure 1).

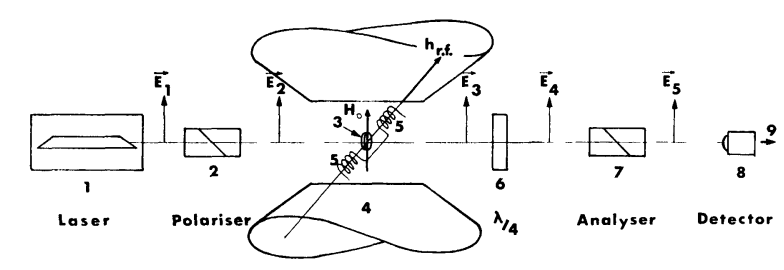

FIG. 3. - Montage expérimental pour obtenir une modulation linéaire de la lumière en utilisant l'anisotropie de la $B$. M. L. ; $1=$ laser, $2=$ polariseur, $3=$ échantillon, $4=$ électroaimant, $5=$ bobine de modulation, $6=$ lame $\lambda / 4,7=$ analyseur, $8=$ détecteur.

variation $\Delta \varphi_{\mathrm{CM}}(t)$ en variation d'intensité lumineuse d'un faisceau laser proportionnelle à $\Delta \alpha(t)$. On peut donc obtenir une modulation linéaire de l'intensité d'un faisceau par lumineux utilisation des effets M. O. du $2^{\mathrm{e}}$ ordre.

La figure 4 montre, pour un échantillon de YIG, taillé parallèlement à un plan (110), l'évolution en fonction de $\alpha$ du déphasage Cotton-Mouton statique $\varphi_{\mathrm{CM}}$ (traits discontinus) ainsi que du taux de modulation de l'intensité d'un faisceau laser (trait plein). La valeur du champ magnétique statique $H_{0}$ est de 1000 Oe et la valeur efficace du champ magnétique de modulation est de 100 Oe. On observe sur cette 


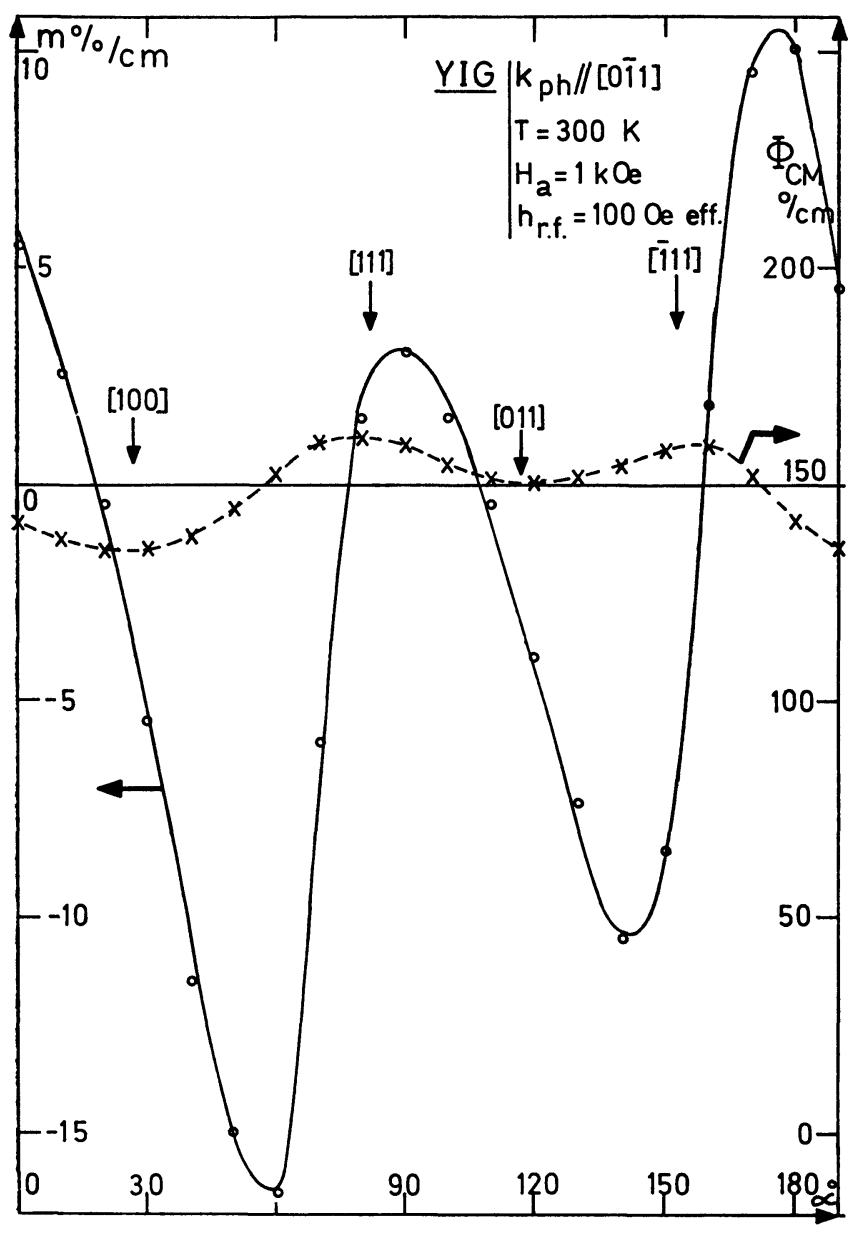

Fig. 4. - Evolution des taux de modulation dans le cas d'un échantillon de $\mathrm{Y}_{3} \mathrm{Fe}_{5} \mathrm{O}_{12}$ d'orientation (110) (-); (- - ) $\varphi_{\mathrm{CM}}$ statique.

figure une très bonne correspondance entre la pente de la courbe statique et le taux de modulation. Le taux de modulation maximum obtenu pour cet échantillon est de \#10\%.

Dans cette première partie, nous avons utilisé la pente $K$ pour illustrer commodément le principe de la modulation décrite dans cet article. Le principe de cette modulation est d'utiliser une variation du déphasage $\varphi_{\mathrm{CM}}$ en fonction de l'orientation de $\mathbf{M}$ par rapport aux axes cristallographiques de l'échantillon, pour obtenir une modulation linéaire d'un faisceau lumineux. En utilisant le modèle phénoménologique des effets M. O. du $2^{\mathrm{e}}$ ordre développé dans la référence [4], on peut déterminer $K$ en fonction des coefficients $M$. O. caractéristiques du matériau M. O. ainsi que des angles d'Euler qui caractérisent l'orientation de $M$. Le calcul se fait en considérant le référentiel trirectangle $X Y Z, Z$ étant la direction de propagation du faisceau lumineux et $\mathbf{X}$ la direction du champ magnétique statique $H_{0}$. Dans ce référentiel, les effets M. O. statiques sont caractérisés par le fait que $\mathbf{M}$ ne comporte qu'une seule composante, la composante $M_{x}(0)$ suivant $\mathbf{X}$. Une variation en fonction du temps de l'orientation de $\mathbf{M}$ corres- pond à l'introduction dans le calcul, développé dans la référence [4], des composantes dépendant du temps $M_{Y}(t)$ et $M_{Z}(t)$.

Dans le cas d'une propagation perpendiculaire à un plan (1 $\overline{1} 0)$, on montre que l'évolution en fonction du temps de $\varphi_{\mathrm{CM}}$ est déterminée par la relation suivante :

$\varphi_{\mathrm{CM}}(t)=\varphi_{0}\left\{1+\frac{B}{A} \cdot \frac{M_{Y}(t)}{M_{X}(0)}+\frac{C}{A} \cdot \frac{M_{Y}^{2}(t)}{M_{X}^{2}(0)}+\cdots\right\}^{1 / 2}$

avec

$$
\begin{gathered}
A=\left\{2 f_{44}-\frac{\Delta f}{2}\left(3 \sin ^{2} \alpha-1\right)\right\}^{2}+ \\
\quad+4 f_{44} \Delta f \sin ^{2} \alpha\left(3 \sin ^{2} \alpha-1\right) \\
B=\frac{1}{2} \Delta f \sin ^{2} \alpha\left\{4 f_{44}\left(12 \sin ^{2} \alpha-5\right)+\right. \\
\left.+3 \Delta f\left(3 \sin ^{2} \alpha-1\right)\right\} \\
C=\left\{8 f_{44}^{2}+6 f_{44} \Delta f\left(3 \sin ^{2} 2 \alpha-1\right)+\right. \\
\left.\quad+\frac{1}{8} \Delta f^{2}\left(27 \sin ^{2} 2 \alpha-8\right)\right\} \\
\varphi_{0}=\frac{1}{2} q_{0} \sqrt{\frac{A}{\varepsilon_{\mathrm{r}}}} M_{x}^{2}(0)
\end{gathered}
$$

d'où

$$
\frac{\Delta \varphi_{\mathrm{CM}}(t)}{\varphi_{0}} \# \frac{B}{2 A} \frac{M_{Y}(t)}{M_{X}(0)}+\frac{C}{2 A} \frac{M_{Y}^{2}(t)}{M_{X}^{2}(0)} .
$$

On vérifie que, au premier ordre, la relation (5) correspond bien à la configuration expérimentale qui a permis de mettre en évidence la modulation présentée dans la figure 4.

On peut montrer que dans le cas d'une propagation perpendiculaire à un plan (111), le déphasage statique est isotrope [4] et a pour valeur :

$$
\varphi_{\mathrm{CM}}(0)=\frac{1}{2 \sqrt{\varepsilon_{\mathrm{r}}}} q_{0}\left\{2 f_{44}+\frac{1}{3} \Delta f\right\} M^{2} .
$$

Alors qu'en présence d'une variation dynamique de l'aimantation, on obtient une composante $\Delta \varphi_{\mathrm{CM}}(t)$ donnée par:

$\Delta \varphi_{\mathrm{CM}}(t) \# \varphi_{0}\left\{1-\frac{D}{2 E} \frac{M_{Z}(t)}{M_{X}(0)}+\frac{M_{Y}^{2}(t)}{M_{X}^{2}(0)}+\cdots\right\}$

avec

$$
\begin{aligned}
D & =2 f_{44}+\frac{1}{3} \Delta f \\
E & =\frac{1}{3} \Delta f \sqrt{2} \sin 3 \alpha \\
\varphi_{0} & =\frac{1}{2} q_{0} \sqrt{\frac{D}{\varepsilon_{\mathrm{r}}}} M_{X}^{2}
\end{aligned}
$$


où $\alpha$ est l'angle que fait $\mathbf{M}$ avec une direction [110] du plan.

Dans le cas présent, on observe qu'une modulation linéaire est obtenue avec un champ de modulation parallèle à la direction de propagation de la lumière (contrairement au cas précédent). La figure 5 montre le taux de modulation obtenu avec une plaquette de TbIG taillée parallèlement à un plan (111) dans la configuration décrite ci-dessus.

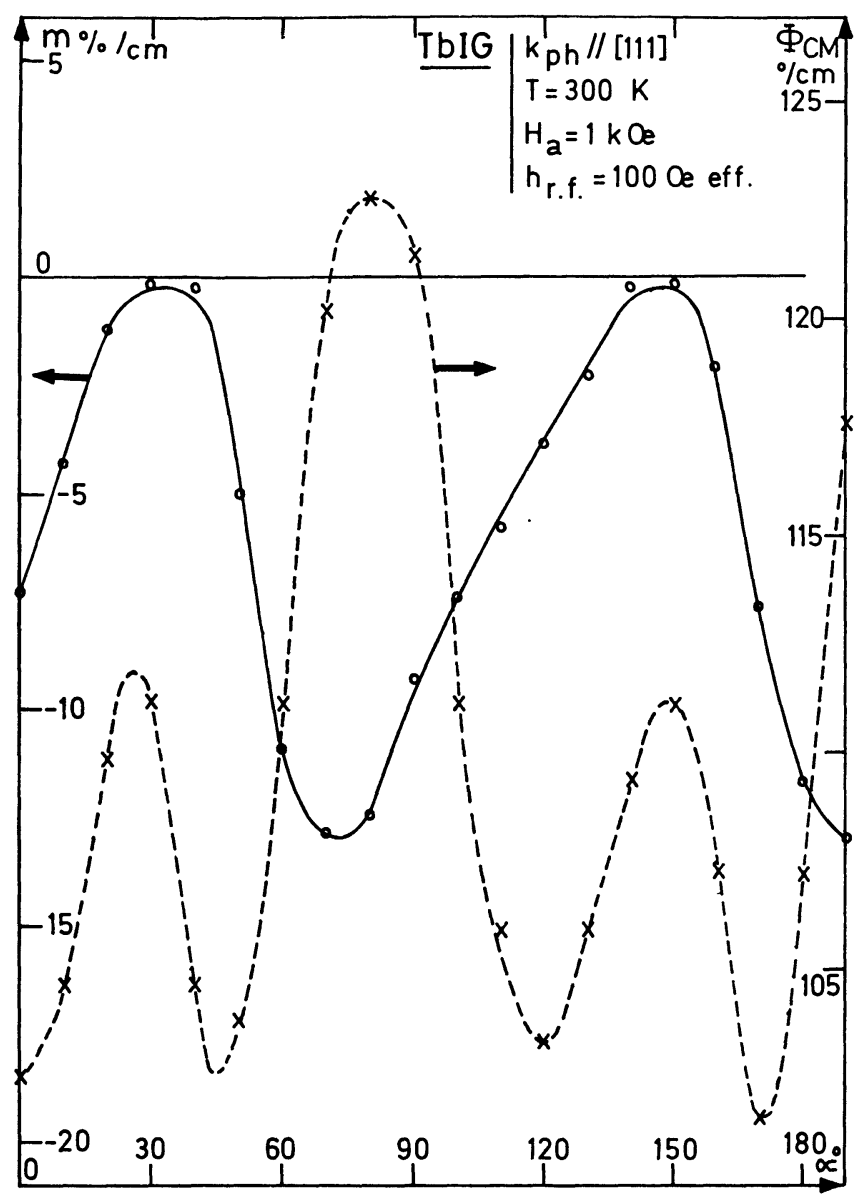

Fig. 5. - Evolution du taux de modulation dans le cas d'un échantillon de TbIG d'orientation (111) (courbe - - ).

On observe que l'évolution du taux de modulation vérifie bien la relation (7) et en particulier la symétrie d'ordre 3. Le taux de modulation obtenu avec le TbIG est de l'ordre de $15 \%$ pour un champ $H_{0}$ de 1000 Oe et un champ $h_{\text {rf }}$ de 100 Oe.

2.2 CRISTAL RHOMboÉDRIQUE (CAS DE L'HÉMATITE $\left.\alpha-\mathrm{Fe}_{2} \mathrm{O}_{3}\right)$. - L'hématite $\alpha-\mathrm{Fe}_{2} \mathrm{O}_{3}$ est un cristal rhomboédrique [7]. Au-dessus de la température de Morin $\left(T_{\mathrm{M}} \neq 269 \mathrm{~K}\right)$, l'hématite est un faible ferromagnétique : l'aimantation spontanée est dans le plan perpendiculaire à l'axe $c$ [8] qui représente un plan de facile aimantation alors que l'axe $c$ est une direc-

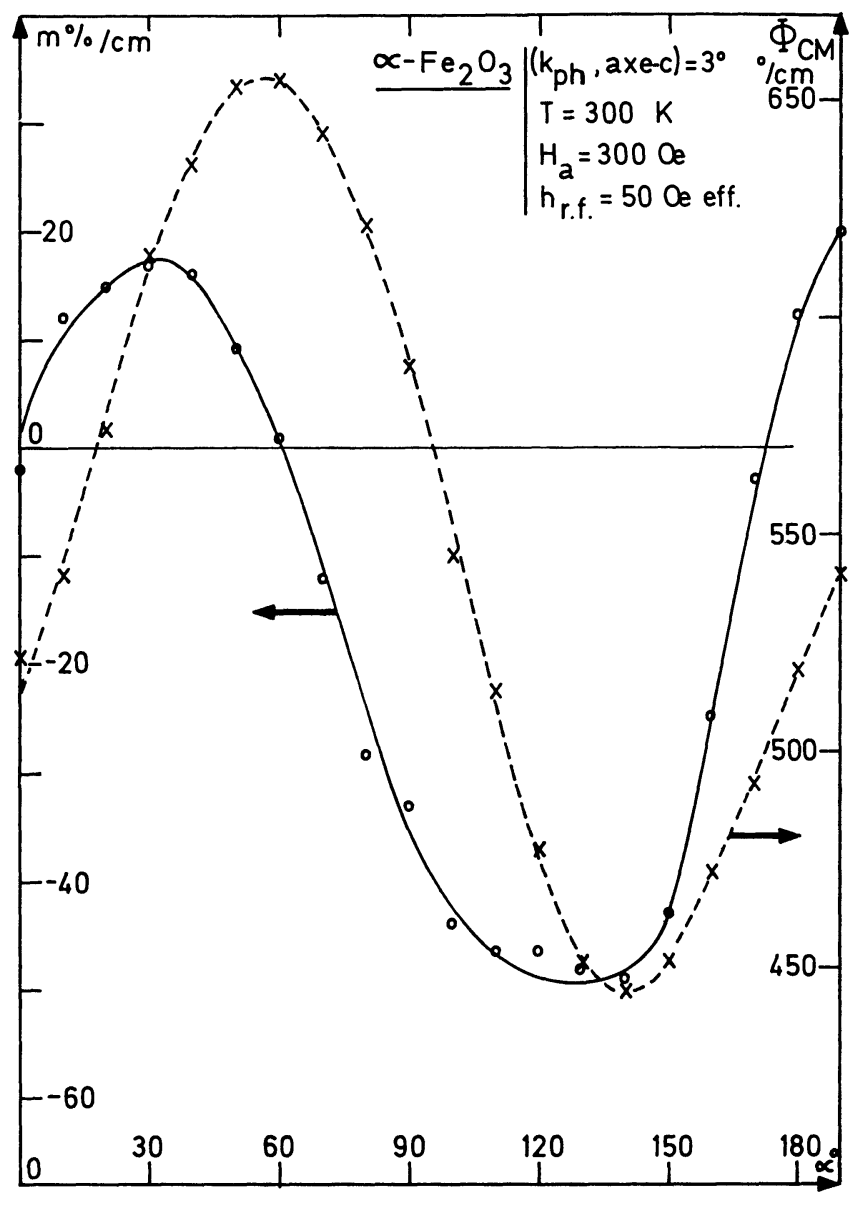

Fig. 6. - Evolution du taux de modulation produit par un échantillon de $\alpha \mathrm{Fe}_{2} \mathrm{O}_{3}$ taillé perpendiculairement à l'axe $c$; $\lambda=1,15 \mu, T=300 \mathrm{~K}, H_{0}=300 \mathrm{Oe}(-)$.

tion de difficile aimantation, avec une anisotropie de l'ordre de $30 \mathrm{kOe}$.

Nous avons mesuré l'effet Cotton-Mouton statique et la modulation dans un disque mince de $\alpha-\mathrm{Fe}_{2} \mathrm{O}_{3}$ taillé sensiblement parallèle au plan de base, dans la configuration expérimentale de la figure 3. L'effet Cotton-Mouton statique présente une anisotropie d'ordre 2 et l'on peut utiliser cet effet pour obtenir une modulation linéaire de la lumière; les résultats obtenus sont portés sur la figure 6. La modulation $(50 \% / \mathrm{cm})$ est obtenue avec un champ de saturation de 300 Oe et un champ de modulation de 50 Oe eff. (plus faible que pour les grenats). Malheureusement l'absorption dans $\alpha-\mathrm{Fe}_{3} \mathrm{O}_{2}$ est de l'ordre de $8 \mathrm{~dB} / \mathrm{mm}$ à $1,15 \mu$. Le modèle théorique de ces effets est en cours d'élaboration.

3. Conclusion. - Nous avons montré théoriquement et expérimentalement la possibilité d'obtenir une modulation linéaire de la lumière en utilisant l'anisotropie des effets magnéto-optiques du $2^{\mathrm{e}}$ ordre (effet Cotton-Mouton) dans les cristaux magnétiques. 


\section{Bibliographie}

[1] LeCRAw, R. C., IEEE Trans. Magn. 2 (1966) 304.

[2] CoOper, R. W., PAGF, J. L., Radio Electron. Eng. 39 (1970) 302.

[3] Pisarev, R. W., Sinit, I. G., Kolpakova, N. N., YakoLEV, Y. M., JETP 33 (1971) 1175.

[4] Tran Khanh Vien, Thèse $3^{\mathrm{e}}$ cycle Paris 1975. [5] Tran Khanh Vien, Jamet, J. P., Legall, H., Krishnan, R.,
Opt. Commun. 13 (1975) 70.

[6] JAMet, j. P., Thèse Paris 1973.

[7] Pauling, L., Hendricks, S. B., J. Am. Chem. Soc. 47 (1925) 781.

[8] Shull, C. G., Strauser, W. A., Wollan, E. O., Phys. Rev. 83 (1951) 333. 